\title{
Wenn Geschlechtsverkehr nicht möglich ist: Vorstellung eines internetbasierten Behandlungsprogramms für Genito-Pelvine Schmerz-Penetrationsstörung mit Falldarstellung
}

\author{
Anna-Carlotta Zarski ${ }^{a, b} \quad$ Matthias Berking ${ }^{a} \quad$ Wiebke Hannig $^{c}$ David Daniel Ebert ${ }^{a}$ \\ a Lehrstuhl für Klinische Psychologie und Psychotherapie, Friedrich-Alexander-Universität Erlangen-Nürnberg, Erlangen, Deutschland; \\ bInstitut für Psychologie, Leuphana Universität Lüneburg, Lüneburg, Deutschland; \\ ${ }^{\mathrm{c}}$ Klinische Psychologie und Psychotherapie, Philipps-Universität Marburg, Marburg, Deutschland
}

\section{Schlüsselwörter}

Genito-Pelvine Schmerz-Penetrationsstörung ·

Sexuelle Funktionsstörung · Kognitive Verhaltenstherapie

\section{Zusammenfassung}

Hintergrund: Schwierigkeiten, Geschlechtsverkehr zu haben trotz bestehenden Wunsches, stellen für Frauen mit Genito-Pelviner Schmerz-Penetrationsstörung (GPSPS) eine große Belastung dar. Die Verfügbarkeit spezifischer Therapieangebote für sexuelle Funktionsstörungen bei Frauen ist begrenzt und existierende Therapiemöglichkeiten werden oftmals aus Schamgefühlen nicht in Anspruch genommen. Internetbasierte Behandlungsansätze können einen niedrigschwelligen, anonymen, zeit- und ortsunabhängigen spezialisierten Therapiezugang ermöglichen. Bislang liegt noch kein wissenschaftlich evaluiertes Programm für die Behandlung von GPSPS vor. Das Ziel der vorliegenden Arbeit ist es deshalb, den Therapieleitfaden eines neu entwickelten internetbasierten, begleiteten Behandlungsprogramms für GPSPS (Paivina-Care) vorzustellen sowie exemplarisch die Akzeptanz und Zufriedenheit und den subjektiven Nutzen anhand eines Fallberichtes darzustellen. Methodik: Das Rational und der Therapieleitfaden des Behandlungsprogramms werden vorgestellt. Die Falldarstellung nach der Case Reporting (CARE)-Leitlinie präsentiert eine Patientin mit erfolgreichem Behandlungsverlauf sowie qualitative und quantitative Erfolgsmaße. Ergebnisse: Die Kasuistik zeigt, dass das kognitiv-verhaltenstherapeutische Programm basierend auf Schmerz- und Sexualtherapie unter Einbezug des Partners die GPSPS bei der vorgestellten Patientin erfolgreich behandeln konnte und mit hoher Therapiezufriedenheit Schmerzen und sexualitätsbezogene Ängste und negative Kognitionen reduzieren sowie Geschlechtsverkehr ermöglichen konnte. Derzeit erfolgt eine randomisiert-kontrollierte Wirksamkeitsuntersuchung an 200 Frauen mit GPSPS. Schlussfolgerung: Das internetbasierte Behandlungsprogramm bietet Frauen mit GPSPS eine flexible Behandlungsmöglichkeit, die sie anonym, zeit- und ortsunabhängig nutzen können.

(C) 2018 S. Karger GmbH, Freiburg

\section{Keywords}

Genito-pelvic pain/penetration disorder .

Sexual dysfunction · Cognitive-behavioral therapy

\section{Summary}

Internet-Based Treatment for Genito-Pelvic Pain/Penetration

Disorder: A Case Report

Background: Difficulties in having sexual intercourse, despite the explicit desire to do so, can lead to high disease burden for women with genito-pelvic pain/penetration disorder (GPPPD). Effective treatment is limited, and only few women actually seek help, often due to feelings of shame. Internet interventions provide anonymous, low-threshold access to specialized treatment, independent of time and place. This study presents the therapy manual of a newly developed guided internet intervention for GPPPD (PaivinaCare) and discloses acceptance, satisfaction, and usefulness by exemplifying with a case report. Methods: The rationale and treatment manual are presented. The case report follows the case reporting (CARE) guidelines and presents a patient with successful treatment course as well as qualitative and quantitative treatment outcomes. Results: A cognitive-behavioral program based on pain management and sex therapy including the partner can be a successful approach to treat GPPPD showing high acceptance and treatment satisfaction. It is currently being evaluated in a randomized-controlled trial of 200 women with GPPPD. Conclusion: This internet intervention offers women with GPPPD a flexible therapy of all symptom dimensions.

\section{KARGER}

(c) 2018 S. Karger GmbH, Freiburg
Dipl.-Psych. Anna-Carlotta Zarski 


\section{Einleitung}

Für Frauen mit Genito-Pelviner Schmerz-Penetrationsstörung (GPSPS) stellen Schwierigkeiten oder die Unmöglichkeit, Geschlechtsverkehr zu haben, obwohl sie es sich wünschen, eine große Belastung und Einschränkung der Lebensqualität dar [Arnold et al., 2006]. Trotz des hohen Leidensdrucks existieren im Vergleich zu anderen psychosomatischen Störungsbildern nur wenige spezialisierte Behandlungsangebote für sexuelle Funktionsstörungen im Allgemeinen und Schmerz-Penetrationsstörungen im Speziellen [Moreira et al., 2005]. Darüber hinaus stellen Schamund Schuldgefühle hinsichtlich sexueller Beschwerden oftmals eine große Barriere für die Inanspruchnahme einer Therapie dar [Nguyen et al., 2013]. Schamgefühle und geringes Störungswissen hemmen überdies oftmals auch Behandelnde, sexuelle Funktionsstörungen im Rahmen von Routineuntersuchungen zu explorieren und zu behandeln [Reinecke et al., 2006].

Die Diagnose der GPSPS entstand im Zuge der Entwicklung der 5. Edition des Diagnostischen und Statistischen Manuals Psychischer Störungen (DSM-5) und vereint die revidierten Diagnosen von Dyspareunie und Vaginismus. Die Zusammenführung beider Störungsbilder in der GPSPS-Diagnose resultierte aus großen Überschneidungen der Symptombilder und Schwierigkeiten ihrer differenzialdiagnostischen Abgrenzung [Carvalho et al., 2012].

Die GPSPS ist im DSM-5 durch 1) Schwierigkeiten bei der vaginalen Penetration während des Geschlechtsverkehrs, 2) vulvovaginale oder Beckenschmerzen während oder bei Versuchen vaginaler Penetration, 3) Angst vor vaginalen Schmerzen oder vaginaler Penetration oder 4) Verkrampfung oder Anspannung der Beckenbodenmuskulatur während versuchter vaginaler Penetration gekennzeichnet [American Psychiatric Association, 2013]. Für eine Diagnosestellung muss mindestens eines der oben genannten Kernsymptome über mindestens 6 Monate bestehen und klinisch bedeutsames Leiden verursachen. Die GPSPS kann als lebenslang (seit Beginn sexueller Aktivität bestehend) klassifiziert werden oder als erworben, wenn die Beschwerden nach einem Zeitraum sexueller Funktionsfähigkeit aufgetreten sind.

Die Prävalenz von GPSPS ist aufgrund der Neuartigkeit der DSM-5-Diagnose noch nicht empirisch untersucht. In Abhängigkeit z.B. von unterschiedlichen Diagnosekriterien variieren die Prävalenzraten in der Allgemeinbevölkerung zwischen 3.0\% und 21.1\% für Dyspareunie und 0.4\% und 6.6\% für Vaginismus [Latthe et al., 2006; Christensen et al., 2011; Peixoto und Nobre, 2015].

Die GPSPS sowie assoziierte Krankheitsbilder wie die vaginalen Schmerzstörungen Vulvodynia und Vestibulodynia haben eine Vielzahl negativer Auswirkungen auf die physische und psychische Gesundheit sowie die Paarbeziehung. Einhergehend mit der GPSPS liegen häufig andere sexuelle Dysfunktionen vor, wie verminderte sexuelle Lust und Erregbarkeit sowie reduzierte sexuelle Aktivität [Farmer und Meston, 2007]. Oftmals sind auch gynäkologische Untersuchungen und die Nutzung von Tampons nicht möglich. Viele betroffene Frauen berichten auch von einem beeinträchtigten Körper- und Selbstbild als Frau [Pazmany et al., 2013]. Komorbid können Depression und Angststörungen auftreten [Khandker et al., 2011].
Die Ätiologie der GPSPS ist multifaktoriell und kann in einem biopsychosozialen Modell konzeptualisiert werden, das physiologische, psychologische, partnerschaftliche und kulturelle Einflussfaktoren berücksichtigt. Die Chronifizierung der GPSPS-Symptomatik kann anhand des Angst-Vermeidungsmodells für chronische Schmerzen [Vlaeyen und Linton, 2000] erklärt werden, in dem insbesondere negative penetrationsspezifischen Kognitionen (z.B. Schmerzkatastrophisierung, Überzeugungen genitaler Inkompatibilität), Hypervigilanz, negative Emotionen wie Angst in penetrationsbezogenen Situationen sowie Vermeidung vaginalen Einführens und sexueller Intimität zur Aufrechterhaltung der Symptomatik beitragen [Cherner und Reissing, 2013].

Aus dem dargestellten Angst-Vermeidungsmodell lassen sich Primärziele für verhaltenstherapeutische Interventionen ableiten: Psychoedukation, Umgang mit dysfunktionalen Kognitionen, Schmerzmanagement, graduierte vaginale Exposition, Förderung der Entspannungsfähigkeit und Paarübungen wie Sensate Focus. Obwohl die einzelnen Therapieelemente einen festen Bestandteil in den wenigen existierenden Behandlungsangeboten für weibliche sexuelle Funktionsstörungen darstellen, existiert bislang nur eine geringe Anzahl methodisch hochwertiger Evaluationsstudien zu deren Wirksamkeit. Psychotherapeutische Formate zur Behandlung von Dyspareunie und Vaginismus umfassen therapeutengeleitete vaginale Einführungsübungen im Praxissetting, kognitivbehaviorale Gruppentherapie oder begleitete bibliotherapeutische Selbsthilfe [Van Lankveld et al., 2006; Bergeron et al., 2016; Brotto et al., 2015b; Günzler und Berner, 2012; Lester et al., 2015]. Studienergebnisse zeigen eine signifikante Zunahme vaginaler Penetrationsfähigkeit, des sexuellen Funktionsniveaus sowie eine Reduktion vaginaler Schmerzen [Ter Kuile et al., 2009].

Traditionelle Behandlungsangebote vor Ort weisen jedoch den Nachteil einer geringeren Reichweite und zeitlichen Gebundenheit auf [Andrade et al., 2014]. Außerdem wirken Schamgefühle aufseiten der Patientinnen in vielen Fällen einer Inanspruchnahme entgegen. Internetbasierte Ansätze weisen in der Behandlung sexueller Funktionsstörungen die Vorteile auf, dass sie 1) zeit- und ortsunabhängig verfügbar sind und somit eine höhere Versorgungsreichweite haben, 2) anonym zugänglich sind, 3) betroffene Frauen aufgrund einer niedrigeren Hemmschwelle der Inanspruchnahme in einem früheren Symptomstadium erreichen können, 4) Frauen ansprechen, die sonst keine Behandlung in Anspruch nehmen würden, und 5) Patientinnen die Möglichkeit geben, in ihrem eigenen Tempo zu arbeiten. Internetbasierte Therapieangebote haben sich bereits in der Behandlung verschiedener sexueller Funktionsstörungen bei Frauen und Männern als wirksam erwiesen [Andersson et al., 2011].

Bislang liegt noch kein wissenschaftlich evaluiertes Behandlungsprogramm für GPSPS vor. Das Ziel der vorliegenden Arbeit ist es deshalb, den Therapieleitfaden eines neu entwickelten internetbasierten Behandlungsprogramms für GPSPS vorzustellen. Anhand einer ausführlichen Kasuistik einer Patientin, die das Programm erfolgreich durchlaufen hat, sollen exemplarisch die Akzeptanz und Zufriedenheit der Intervention und ihr subjektiver Nutzen dargestellt werden. 


\section{Darstellung des Therapieleitfadens}

\section{Therapieformat}

Das Behandlungsprogramm für GPSPS wurde basierend auf bisherigen Forschungsergebnissen zu Vaginismus und Dyspareunie konzipiert und beinhaltet Elemente kognitiv-verhaltenstherapeutischer Schmerz- und Sexualtherapie. In einer Pilotstudie wurde die Vorgängerversion des Programms für Frauen mit Vaginismus evaluiert, auf der das hier präsentierte internetbasierte Behandlungsprogramm für GPSPS aufbaut [Zarski et al., 2017]. Die Behandlung erfolgt im Einzelsetting unter Einbezug des Partners. Gezielte Interventionen umfassen Psychoedukation, kognitive Umstrukturierung, bewertungsfreies Wahrnehmen, Muskel- und Atementspannung, Entspannung des Beckenbodens, schmerzbewältigende Aufmerksamkeitsfokussierung, Körperexposition und genitale Selbstexploration, Förderung sexueller Lust und Erregung, graduelle Exposition mit Fingern und Vaginaldilatoren, Sensate Focus, Einführungsübungen des Penis des Partners, Vorbereitung des Geschlechtsverkehrs und Rückfallprophylaxe.

Die einzelnen Behandlungsbausteine verteilen sich auf insgesamt 8 Module, die die Patientinnen in festgelegter Reihenfolge durchlaufen. Vier Wochen nach Abschluss der Module folgt ein weiteres Auffrischungsmodul. Die Bearbeitungszeit für 1 Modul beträgt zwischen 45 und 60 min. Empfohlen wird, 1 Modul pro Woche zu absolvieren.

Die Module bestehen aus textbasierten Informationen, Erfahrungsberichten von Beispielpatientinnen sowie interaktiven Elementen wie Übungen für den Alltag, schriftlichen Einträgen, Wissensquiz, MP3-Audiodateien, Videos und Arbeitsblättern zum Herunterladen. Das Programm ist adaptiv, sodass sich der Inhalt durch verschiedene Antwort- und Auswahlmöglichkeiten an die jeweiligen Bedürfnisse der Patientinnen anpasst. Zusätzlich können Patientinnen ein Online-Tagebuch nutzen, in dem sie über eine Kalenderfunktion grafisch visualisierbare Therapiefortschritte einschätzen und hilfreiche Strategien dokumentieren. Tagebucheinträge können als Smartphone-App auch unmittelbar in relevanten Alltagssituationen vorgenommen werden. Die Übungen im Alltag sind zentraler Programmbestandteil, um die direkte Anwendung neu erlernter Strategien zu fördern.

\section{Psychologische Begleitung}

Die Patientinnen werden im Programmverlauf durch eine Betreuerin begleitet, die innerhalb von $48 \mathrm{~h}$ eine schriftliche Rückmeldung auf absolvierte Module gibt. Der Betreuungsschwerpunkt liegt in der Validierung von Trainingserfolgen, der Motivierung der Patientinnen zu regelmäßiger Übungsdurchführung, der Unterstützung im Umgang mit aufkommenden Schwierigkeiten und der Beantwortung von Fragen. Die Betreuerin erinnert die Patientinnen außerdem an die Modulabsolvierung, wenn ein Modul nicht innerhalb von 7 Tagen bearbeitet wurde. Bei Bedarf können individuelle zeitliche Vereinbarungen getroffen werden.
Die Betreuerinnen sind Psychologiestudentinnen, die eine umfassende Schulung zu Störungsbild, GPSPS-Behandlung, Nutzung der Online-Plattform und der manualbasierten schriftlichen Rückmeldung an die Patientinnen erhalten haben. Sie wurden kontinuierlich durch eine Psychologische Psychotherapeutin in Ausbildung sowie eine approbierte Psychologische Psychotherapeutin supervidiert.

\section{Therapiebausteine}

\section{Modul 1: Wissenswertes}

Psychoedukation I

Das Programm beginnt mit einer umfassenden Psychoedukation zum Symptombild, zur Prävalenz und zum Entstehungs- und Risikofaktorenmodell der GPSPS. Die Patientinnen haben die Möglichkeit, sich in einem Quiz spielerisch mit verschiedenen GPSPS-Mythen (z.B. «Der Penis kann in der Vagina stecken bleiben») auseinanderzusetzen sowie die individuelle Entstehungsgeschichte ihrer GPSPS aufzuschreiben. Tabelle 1 zeigt die Module und Therapiebausteine des Behandlungsprogramms für GPSPS im Überblick.

\section{Förderung der Therapieadhärenz und}

Selbstwirksamkeitserwartungen

Zur Förderung der Therapie- und Übungsmotivation formulieren die Patientinnen ihre persönlichen Motivationsgründe für die Behandlung sowie individuelle Therapieziele, die im Programmverlauf wiederholt aufgegriffen werden. Die Bedeutsamkeit des regelmäßigen Übens im Alltag wird als wichtigster Wirkfaktor für therapeutische Veränderungen hervorgehoben. Um die Patientinnen hierbei zu unterstützen, können sie Trainingszeiten gezielt in ihren Online-Wochenplan integrieren und Bewältigungsideen für mögliche Hindernisse und Rückschläge bei der Übungsdurchführung vorbereiten. Darüber hinaus werden die Patientinnen in das Konzept der effektiven Selbstunterstützung eingeführt und zu Selbstbelohnungen angeleitet.

\section{Einbezug des Partners}

Die Patientinnen werden zum Abschluss des ersten Moduls dazu ermutigt, das Gespräch mit ihrem Partner bezüglich der GPSPS-Therapie, der Rolle des Partners in der Behandlung und beidseitiger emotionaler Bedürfnisse zu suchen. Unterstützend werden Tipps für gute partnerschaftliche Kommunikation vorgestellt.

\section{Modul 2: Ausstieg aus dem GPSPS-Teufelskreis}

\section{Psychoedukation II}

Im zweiten Teil der Psychoedukation erweitern die Patientinnen ihr individuelles GPSPS-Erklärungsmodell um das Angst-Vermeidungsmodell. An einer selbstgewählten GPSPS-relevanten Situation können sie situationsspezifische Gedanken, Gefühle, Körperreaktionen und Verhaltensweisen festhalten und deren Zusammenhänge 
Tab. 1. Module und Therapiebausteine des Behandlungsprogramms für GPSPS

\begin{tabular}{|c|c|c|}
\hline Modul & & Therapiebausteine \\
\hline 1 & Wissenswertes & $\begin{array}{l}\text { Psychoedukation I } \\
\text { Förderung der Therapieadhärenz } \\
\text { Einbezug des Partners in das Training }\end{array}$ \\
\hline 2 & $\begin{array}{l}\text { Ausstieg aus dem GPSPS } \\
\text { Teufelskreis }\end{array}$ & $\begin{array}{l}\text { Psychoedukation II } \\
\text { kognitive Umstrukturierung } \\
\text { bewertungsfreies Wahrnehmen }\end{array}$ \\
\hline 3 & $\begin{array}{l}\text { Lockerlassen und erstes } \\
\text { Einführen }\end{array}$ & $\begin{array}{l}\text { Muskel- und Atementspannung; gezielte Beckenbodenentspannung } \\
\text { schmerzbewältigende Aufmerksamkeitsfokussierung } \\
\text { graduelles vaginales Einführen mit Fingern }\end{array}$ \\
\hline 4 & Sich selbst entdecken & Körperexposition und genitale Selbstexploration \\
\hline 5 & Vaginaltraining I & $\begin{array}{l}\text { Förderung sexueller Lust und Erregung } \\
\text { graduelles vaginales Einführen mit Dilatoren I } \\
\text { graduelles vaginales Einführen mit Dilatoren seitens des Partners I } \\
\text { Sensate Focus I }\end{array}$ \\
\hline 6 & Vaginaltraining II & $\begin{array}{l}\text { graduelles vaginales Einführen mit Dilatoren II } \\
\text { graduelles vaginales Einführen mit Dilatoren seitens des Partners II } \\
\text { Sensate Focus II }\end{array}$ \\
\hline 7 & Geschlechtsverkehr & Einführungsübungen des Penis des Partners und Vorbereitung des Geschlechtsverkehrs \\
\hline 8 & Reflexion und Ausblick & Zukunftsplan und Rückfallprävention \\
\hline 9 & Auffrischung & Reaktivierung gelernter Behandlungsinhalte \\
\hline
\end{tabular}

erarbeiten. Das Angst-Vermeidungsmodell wird als Rational für die weiteren Behandlungsbausteine wiederholt aufgegriffen.

\section{Kognitive Umstrukturierung}

Anknüpfend an das Angst-Vermeidungsmodell werden die Patientinnen in der kognitiven Umstrukturierung angeleitet, in einem Gedankenprotokoll ihre Gedanken zu beobachten und mithilfe kognitiver Techniken wie dem Perspektivwechsel oder Realitätstest zu hinterfragen. Anschließend formulieren sie hilfreiche Alternativgedanken, um ihre Bewertung hinsichtlich des vaginalen Einführens zu verändern. Abschließend werden Strategien vorgestellt, wie die hilfreichen Gedanken im Alltag erinnert werden können (z.B. Notiz auf Nachttisch). Als Hausaufgabe üben die Patientinnen die Anwendung des Gedankenprotokolls und der hilfreichen Gedanken in sexuellen und nichtsexuellen Situationen.

\section{Bewertungsfreies Wahrnehmen}

Zum Erlernen des Umgangs mit automatischen negativen Gedanken und Gefühlen wird außerdem die Methode des bewertungsfreien Wahrnehmens vorgestellt [Brotto et al., 2015a]. Hierbei werden die Patientinnen angeleitet, ihre Gedanken und Gefühle zu beobachten, ohne sie zu bewerten oder zu unterdrücken. Bewertungsfreies Wahrnehmen wird ebenfalls in sexuellen und nichtsexuellen Situationen geübt.

\section{Modul 3: Lockerlassen und erstes Einführen}

\section{Muskel- und Atementspannung}

Mit einer Audiodatei zu progressiver Muskelentspannung (PMR) in Kombination mit Atementspannung erlernen die Patientinnen eine gezielte Ganzkörper-Entspannungsmethode [Jacobson, 1938]. Sie üben die PMR zunächst in einer Lang-, später in einer
Kurzversion. Nach dem Prinzip der Muskelan- und -entspannung trainieren die Patientinnen anschließend anhand eines Lehrvideos die gezielte Wahrnehmung und Entspannung der Beckenbodenmuskulatur.

\section{Schmerzbewältigende Aufmerksamkeitsfokussierung}

In einem Video wird zunächst die Funktionsweise und multifaktorielle Entstehung von Schmerzen sowie die Unterscheidung zwischen akuten und chronischen Schmerzen erläutert. Patientinnen können daraufhin individuelle Einflussfaktoren ihrer Schmerzen identifizieren. In der Aufmerksamkeitsfokussierung werden sie mit einer Audiodatei angeleitet, in einer Imagination zunächst ihr genito-pelvines Schmerzzentrum zu lokalisieren, sich den Schmerz vorzustellen und ihn bildlich zu beschreiben. Daraufhin wird die Defokussierung des Schmerzes durch gezielte Lenkung der Aufmerksamkeit auf z.B. Umgebungsgeräusche, positives Erleben und den Atem geübt und zur Veränderung der Schmerzqualität eine Transformation des Schmerzbildes angeleitet (z.B. Veränderung der Form, Farbe und Temperatur des Schmerzbildes) [Dillworth und Jensen, 2010].

\section{Graduelles vaginales Einführen mit Fingern}

Die vaginalen Einführungsübungen basieren auf dem Prinzip gradueller Exposition und werden in Kombination mit der Muskel-, Atem- und Beckenbodenentspannung durchgeführt. Das vaginale Einführen beginnt mit dem Selbsteinführen von einem und zwei Fingern. Um Kontrollerleben und Handlungsfähigkeit während des vaginalen Einführens zu stärken, nutzen die Patientinnen eine schrittweise Anleitung zum Herunterladen und werden dazu angeregt, eigene Rituale zur Übungsvorbereitung zu entwickeln (z.B. angenehme Umgebung schaffen, potenzielle Störvariablen wie Handy ausschalten). 


\section{Modul 4: Sich selbst entdecken}

Körperexposition und genitale Selbstexploration

Die Körperexposition und genitale Selbstexploration beginnt mit der Illustration und Erläuterung der weiblichen Genitalien, der Anatomie des Beckenbodens und der physiologischen Prozesse während der sexuellen Erregung und des Geschlechtsverkehrs. In der anschließenden Spiegelübung betrachten und ertasten die Patientinnen ihre Vagina vor einem Spiegel unter Anwendung bewertungsfreier Wahrnehmung. In weiteren Kurzübungen zur Stärkung der positiven Körperwahrnehmung und des Selbstwertes lenken die Patientinnen gezielt den Fokus auf Körperteile, die sie an sich mögen, identifizieren eigene Stärken und Erfolge und entwickeln Strategien für selbstfürsorgliches Verhalten.

\section{Sensate Focus I}

Sensate-Focus-Übungen dienen der gegenseitigen sinnlichen Körperwahrnehmung zwischen Patientin und Partner, um mit dem eigenen Körper und dem Körper des Partners einschließlich erogener Zonen vertrauter $\mathrm{zu}$ werden [Masters und Johnson, 1986]. Zu Beginn werden die Regeln des schrittweisen Vorgehens vorgestellt, um Performanzdruck und Angst bei sexueller Intimität abzubauen. Die Partner berühren und massieren sich gegenseitig 1) zunächst vollständig bekleidet ohne Einbezug der Genitalien, anschließend in Unterwäsche, 2) mit Berührung der Genitalien, aber ohne das Ziel, sexuelle Erregung hervorzurufen, und 3) mit Berührung der Genitalien, die auch zu sexueller Erregung führen darf. Während der Übungen nehmen beide Partner sowohl die aktive als auch die passive Rolle des Berührens ein. Die Paare werden außerdem dazu angehalten, ihre Gefühle und Empfindungen miteinander zu teilen, um zu erfahren, was ihnen selbst und ihrem Partner positive Empfindungen und Lust bereitet.

\section{Modul 5: Vaginaltraining I}

\section{Förderung sexueller Lust und Erregung}

Zur Förderung sexueller Lust und Erregung wird sexuelle Selbststimulation in Form von Masturbationstechniken thematisiert, die als Hausaufgabe während der vaginalen Einführungsübungen ausprobiert werden können.

\section{Graduelles vaginales Einführen mit Dilatoren I}

Die graduelle Exposition wird mit dem Selbsteinführen von Dilatoren, d.h. ein medizinisches Gerät, das im Sinne einer systematischen Desensibilisierung zum kontrollierten vaginalen Einführen unter begleiteter Anwendung von Entspannungstechniken verwendet wird, in verschiedenen Größen fortgeführt, beginnend mit dem kleinsten und abschließend mit dem größten Dilator, entsprechend der durchschnittlichen Penisgröße. Der Übergang zum nächstgrößeren Dilator wird empfohlen, wenn Patientinnen die vorangegangene Größe wiederholt problemlos einführen können. Eine schrittweise Anleitung thematisiert außerdem Übungsvorbereitungen, verschiedene Sitzpositionen und Tipps zum Umgang mit Schwierigkeiten. Zusätzlich wird den Patientinnen eine
Langzeitübung angeboten, bei der sie den Dilator bis zu einer Stunde in der Vagina behalten, um den Habituationseffekt zu verstärken.

Graduiertes vaginales Einführen mit Dilatoren seitens des Partners

Die Einführungsübungen mit dem Partner sind ebenfalls schrittweise aufgebaut und beginnen mit dem Einführen von 1) einem und zwei Fingern des Partners, gefolgt von 2) Dilatoren in aufsteigender Größe, während die Frau jeweils die Hand des Partners führt.

\section{Modul 6: Vaginaltraining II}

An dieser Stelle im Programm können die Patientinnen auswählen, ob sie weiterhin mit den kleineren Dilatorengrößen üben oder zum Einführen des größten Dilators übergehen möchten. Weiterhin wird außerdem vaginales Einführen und Sensate Focus mit dem Partner durchgeführt. Darüber hinaus werden konkrete Schritte zur Problemlösung von Übungsschwierigkeiten (z.B. keine Zeit, Einführungsprobleme) anhand von vorgestellten Bewältigungsstrategien (z.B. Übungszeiten in Terminkalender einplanen, Positionswechsel beim Einführen) erarbeitet.

\section{Modul 7: Geschlechtsverkehr}

\section{Einführen des Penis des Partners}

Die Einführungsübungen des Penis des Partners umfassen 1) Berührungen der Vagina mit dem erigierten Penis, ohne dass es zur Penetration kommt, 2) Einführen des erigierten Penis, ohne dass sich der Partner dabei bewegt, und 3) Bewegung des eingeführten Penis in der Vagina.

\section{Modul 8: Reflexion und Ausblick}

Im Abschlussmodul evaluieren die Patientinnen die Wirksamkeit der einzelnen Therapiebausteine, reflektieren ihre Trainingsziele und können sich neue Ziele für die nächsten 4 Wochen setzen. Im Zuge dessen verfassen sie einen Brief an sich selbst zu Wünschen hinsichtlich ihres (Sexual-)Lebens. Mit dem Ziel der Rückfallprävention identifizieren die Patientinnen außerdem Frühwarnzeichen und Rückfallstrategien.

\section{Auffrischungsmodul}

Vier Wochen nach Abschluss des achten Moduls können die Patientinnen mithilfe der Evaluation des Briefes an sich selbst ihre erreichten Ziele reflektieren und Bilanz ziehen. Abschließend haben sie die Möglichkeit, neue Ziele zur Aufrechterhaltung des beschwerdefreien Zustands, zur Bewältigung von Residualsymptomatik oder zur Weiterentwicklung ihres Sexuallebens zu formulieren.

\section{Design der Falldarstellung}

Das Behandlungsprogramm wird derzeit in einer randomisiertkontrollierten Studie mit Wartekontrollgruppe an 200 Patientin- 
nen mit GPSPS evaluiert (genehmigt durch die Ethikkommission Universität Erlangen-Nürnberg, No. 324_15B; registriert im Deutschen Register Klinischer Studien: DRKS00010228). Die Studieneinschlusskriterien umfassen: 1) kein Geschlechtsverkehr in den letzten 6 Monaten aufgrund von GPSPS (Primary Endpoint Questionnaire (PEQ) Item $7 \leq 1$ ) [Van Lankveld et al., 2006], 2) Alter $\geq$ 18 Jahre, 3) Ausschluss medizinisch behandelbarer GPSPS-Ursachen, 4) heterosexuelle Partnerschaft/Übungspartner, 5) Internetzugang, 6) ausreichende Deutschkenntnisse und 7) schriftliche informierte Einwilligung in die Studienteilnahme. Die Ausschlusskriterien sind: 1) Psychose oder dissoziative Symptome, 2) derzeitige oder vergangene posttraumatische Belastungsstörung, 3) derzeitige Substanzabhängigkeit, 4) mittlere oder schwere Depression oder bipolare Störung erfasst mit dem Strukturierten Klinischen Interview für DSM-IV (SKID-I) via Telefon [Wittchen et al., 1997] und 5) derzeit in Behandlung für GPSPS.

Die Behandlungseffektivität und -zufriedenheit werden anhand einer Falldarstellung einer Patientin mit erfolgreichem Behandlungsverlauf veranschaulicht. Hierfür werden schriftliche Eintragungen der Patientin in den Online-Modulen und ihre Angaben in einem qualitativen Telefoninterview nach Therapieabschluss berichtet. Zur Beurteilung von Behandlungserfolg, -akzeptanz und -zufriedenheit werden die quantitativen Angaben der Patientin aus den onlinebasierten Befragungen vor Beginn und nach Abschluss der Behandlung sowie das qualitative Telefoninterview ausgewertet. Die Falldarstellung folgt der Case-Reporting(CARE)-Leitlinie [Gagnier et al., 2013]. Die Patientin willigte schriftlich in die Studienteilnahme, Datenerhebung und -auswertung sowie Erstellung und Veröffentlichung des Fallberichts ein.

Quantitative Erfolgsmaße: Der PEQ wurde zur Erfassung koitaler und nichtkoitaler Penetrationsfähigkeit (7 Items, 4-stufige Likert-Skala) eingesetzt. Der Fear of Sexuality Questionnaire (FSQ; 5 Items, 5-stufige Skala) erhebt die Angst vor Geschlechtsverkehr und nichtkoitaler sexueller Aktivität [Ter Kuile et al., 2013]. Der Vaginal Penetration Cognition Questionnaire (VPCQ; 22 Items, 7-stufige Skala) erfasst positive und negative Kognitionen hinsichtlich vaginaler Penetration [Klaassen und Ter Kuile, 2009]. Der Female Sexual Function Index (FSFI; 19 Items, 7-stufige Skala) erhebt das sexuelle Funktionsniveau [Rosen et al., 2000] und der Partnerschaftsfragebogen (PFB-K; 9 Items, 4-stufige Skala, «Glücks-Items», 6-stufige Skala) erfasst die Partnerschaftsqualität und -zufriedenheit [Kliem et al., 2012]. Potenzielle negative Effekte wurden mit dem Inventory for the Assessment of Negative Effects in Psychotherapy (INEP; 15 Items, 7-stufige und 4-stufige LikertSkalen) gemessen [Ladwig et al., 2014].

\section{Fallbericht}

\section{Vorgeschichte}

Frau Apfel (Name geändert) war zum Zeitpunkt ihrer Teilnahme am Behandlungsprogramm 29 Jahre alt. Die Patientin mit Fachhochschulabschluss sei gemäß eigener Angaben verheiratet und seit 11 Jahren in einer Beziehung mit ihrem Partner. Sie leide seit 12 Jahren unter GPSPS-Symptomen und Geschlechtsverkehr sei ihr noch nie möglich gewesen. Sie gab an, dass medizinisch behandelbare Ursachen der GPSPS gynäkologisch ausgeschlossen worden seien. Missbrauchserlebnisse wurden nicht berichtet. Frau Apfel habe bereits eine ambulante Psychotherapie absolviert, die hinsichtlich ihrer GPSPS-Beschwerden keinen Erfolg gebracht habe. Als Grund für ihre Programmteilnahme führte sie an, ein onlinebasiertes Angebot ansprechend zu finden.

\section{Diagnostik}

Frau Apfel erfüllte die DSM-5-Kriterien für eine GPSPS und berichtete, dass sie und ihr Partner sexuell aktiv seien, aber in den letzten 6 Monaten nicht versucht hätten, den Penis ihres Partners einzuführen, aufgrund von brennenden und ziehenden Schmerzen einhergehend mit starken Ängsten bei Versuchen vaginaler Penetration. Auch falle es ihr schwer, in sexuellen Situationen die Kontrolle abzugeben. Es lagen keine komorbiden psychischen Störungen vor. Ihre Ehe schätzte die Patientin als sehr glücklich ein. Sie beschrieb ihren Partner als sehr unterstützend und gab an, dass sie mit ihm sexuelle Intimität und Lust erleben könne. Ihr Partner gab keine sexuellen Funktionsstörungen an.

\section{Beschreibung der Behandlung}

Die Patientin berichtete, sich in den psychoedukativen Informationen «gut wiedererkennen» zu können und sich im Erleben ihrer Beschwerden «nicht mehr alleine» zu fühlen. Als Einflussfaktoren ihrer GPSPS nannte Frau Apfel, «ein ängstlicher Typ» zu sein und «sich immer viele Gedanken zu machen», Schwierigkeiten, mit Beginn der ersten Menstruation Tampons einzuführen, und die daraus resultierende "Angst zu versagen», eine fehlende Ansprechpartnerin zur Besprechung sexueller Themen in der Pubertät sowie schmerzhafte und abwertende Erfahrungen bei gynäkologischen Untersuchungen.

Frau Apfel profitierte im Verlauf der Behandlung stark von der Erarbeitung konkreter und kleinschrittiger Behandlungsziele und ihrer Therapie- und Änderungsmotive, um sich zu motivieren und auf das Programm «einlassen» zu können. Als Behandlungsziele definierte die Patientin, Geschlechtsverkehr mit ihrem Partner haben zu können, Angst vor Schmerzen zu reduzieren, Umgang mit negativen penetrationsspezifischen Gedanken und es «nicht mehr als persönliches Versagen zu erleben», wenn Geschlechtsverkehr nicht möglich ist. Langfristig wünsche sie sich, auf natürlichem Weg schwanger zu werden. Sie formulierte als Motivationssatz: «Ich möchte mich freier und unbeschwerter fühlen und auf nichts mehr verzichten müssen.» Frau Apfel überlegte sich außerdem zu Behandlungsbeginn konkrete Strategien für regelmäßiges Üben, wie das Einplanen fester Übungszeiten, die Visualisierung der Nützlichkeit der Übungen und gezielte Belohnungen (z.B. in Hängematte lesen).

Als Beispielsituation für die Erstellung ihres individuellen Störungsmodells wählte Frau Apfel einen Untersuchungstermin bei ihrer Gynäkologin. Frau Apfel berichtete folgende Gedanken in dieser Situation: «Werde ich Schmerzen haben?», «Bei allen anderen klappt es, nur bei mir nicht» und «Wird mir etwas peinlich oder unangenehm sein?». Damit einhergehende Gefühle seien Angst (Intensität 7 von 10), Ungewissheit (Intensität 6 von 10) und Ärger/Frustration (Intensität 5 von 10). Ihr Körper reagiere mit Verkrampfungen, steigendem Puls, Herzrasen, Zittern, kalten Händen und Unruhe. Daraufhin sage sie ihrer Gynäkologin, dass sie «keine vaginale Untersuchung möchte».

Anschließend erarbeitete sich Frau Apfel in der kognitiven Umstrukturierung folgende hilfreiche Gedanken für vaginales Einführen und Geschlechtsverkehr: «Mein Körper ist dafür geschaffen», "Auch wenn es etwas weh tun sollte, kann ich damit umgehen, ich brauche nichts zu fürchten» und «Ich kann meinem Partner jederzeit sagen, wenn ich etwas anders möchte, mir etwas zu viel sein sollte». Damit einhergehend berichtete sie deutlich weniger Angst und mehr Zuversicht. Zur Schmerzbewältigung profitierte die Patientin sehr von der kognitiven Umstrukturierung, die ihr geholfen habe, «Unbekanntes nicht gleich als Schmerz einzuordnen, sondern es einfach erstmal als neu und unbekannt zu bewerten».

Die Beckenbodenentspannung empfand Frau Apfel ebenfalls als hilfreich, da sie dabei das Gefühl verspüre, sich «zu lockern und zu öffnen». Das Einführen der Finger sei ihr anschließend problemlos und schmerzfrei möglich gewesen. Gleichzeitig berichtete sie jedoch ein Engegefühl beim Einführen und die Sorge, «sich nicht vorstellen zu können, dass etwas Größeres in sie hineinpasse». Im Rahmen der Körperexposition und genitalen Selbstexploration habe es ihr 
«gutgetan, sich der eigenen Anatomie wieder bewusst zu werden und sich zu entdecken».

Im weiteren Verlauf berichtete die Patientin von Arbeitsstress, der sie von der regelmäßigen Durchführung des Vaginaltrainings abhalte. Es sei ihr aber auch wichtig gewesen, «sich nicht fertigzumachen und zu selbstkritisch und perfektionistisch zu sein», da die Übungen «weiterhin etwas Bestärkendes und Positives bleiben sollten». Frau Apfel führte daraufhin ihre bereits geplanten Übungsstrategien durch (konkrete Terminplanung, Planung ihrem Partner mitteilen für größere Verbindlichkeit, sich kleine Erfolge bewusstmachen). Auch seien die Rückmeldungen und Erinnerungen der Betreuerin bei der Etablierung einer kontinuierlichen Übungsroutine unterstützend gewesen, ebenso wie das Online-Tagebuch mit seiner grafischen Visualisierung des Übungsfortschrittes. Gegen Trainingsende habe sie das Übungstagebuch nicht mehr benötigt.

Als wichtigsten Behandlungsbaustein nannte Frau Apfel das kontinuierliche Vaginaltraining mit unterschiedlichen Dilatorgrößen, die sie zunächst alleine und später mit ihrem Partner in ihrem «eigenen Tempo» eingeführt habe. Die Patientin berichtete, bereits Erfahrungen mit Dilatorenübungen gemacht $\mathrm{zu}$ haben, aber aufgrund von Schwierigkeiten beim Einführen größerer Größen «etwas abgeschreckt» gewesen zu sein und «aufgegeben» zu haben. Im Verlauf des Vaginaltrainings berichtete Frau Apfel, dass es ihr gelungen sei, die kleineren Dilatoren fast täglich erfolgreich einzuführen. Hierbei haben ihr nach eigenen Angaben «feste Übungsstrukturen und Rituale», «Zeit und Ruhe zum Üben» und der schrittweise Aufbau «viel Sicherheit und Kontrolle» gegeben, sodass sie sich «nicht mehr ohnmächtig gegenüber der GPSPS» gefühlt habe.

Die Sensate-Focus-Übungen mit ihrem Partner habe die Patientin ebenfalls als sehr positiv erlebt, da es schön gewesen sei, sich «bewusst Zeit füreinander zu nehmen», sich «zu spüren» und «auch wieder mal etwas anderes kennenzulernen, obwohl man schon recht lange zusammen ist».

Bevor sie mit den Einführungsübungen des Penis ihres Partners begann, entwickelte Frau Apfel vielfältige positive Selbstinstruktionen, wie «Ich kenne meinen Körper inzwischen so gut und weiß, dass ich so gebaut bin, dass ich den Penis aufnehmen kann», "Ich habe viel mit den Vaginaltrainern und auch mit meinem Partner geübt. Ich konnte Sicherheit gewinnen, viele Ängste abbauen». Das Einführen des Penis gelang Frau Apfel beim ersten Übungsversuch problemlos und sie habe den Übergang von den Dilatoren zum Penis entgegen ihrer Befürchtungen als «einfach» empfunden, da dieser «beweglicher, anpassungsfähiger und weicher» sei. Zur Stabilisierung ihrer Trainingserfolge gab Frau Apfel an, weiterhin regelmäßig vaginales Einführen üben, aber auch Flexibilität und Spontanität im Sexualleben mit ihrem Partner aufbauen zu wollen.

\section{Therapieergebnis}

Frau Apfel absolvierte die 8 Therapiemodule innerhalb von 3 Monaten sowie die Auffrischungslektion 4 Wochen später. Nach Abschluss gab sie im primären Erfolgsmaß PEQ an, erfolgreich den Penis ihres Partners einführen zu können. Ebenfalls zeigte sich eine deutliche Schmerzreduktion während des Geschlechtsverkehrs im Skalenwert (SW) Schmerz des FSFI ( $\mathrm{SW}_{\text {prä }}=4,00, \mathrm{SW}_{\text {post }}=2,80$, Range: 0-5). Auch dysfunktionale schmerzbezogene Kognitionen wie «Ich fürchte, dass ich nichts tun kann, um den Schmerz beim vaginalen Einführen zu verändern» $\left(S_{\text {prä }}=14, S_{\text {post }}=1\right.$, Range: 0-30), sowie Angst vor Geschlechtsverkehr (FSQ: $S_{\text {prä }}=10, S_{\text {post }}=5$, Range: 5-15), vor Kontrollverlust (VPCQ: $\mathrm{SW}_{\text {prä }}=6, \mathrm{SW}_{\text {post }}=0$, Range: 0-24) und genitaler Inkompatibilität mit dem Partner (VPCQ: $\mathrm{SW}_{\text {prä }}=9, \mathrm{SW}_{\text {post }}=1$, Range: $0-12$ ) reduzierten sich fast vollständig im VPCQ. Negative selbstwertbezogene Kognitionen («Ich fühle mich schuldig, wenn vaginales Einführen nicht möglich ist») zeigten sich ebenfalls deutlich gemindert (VPCQ: $\mathrm{SW}_{\text {prä }}=18, \mathrm{SW}_{\text {post }}=5$, Range: 0-36). Gleichzeitig nahmen positive Gedanken hinsichtlich Geschlechtsverkehr zu $\left(\mathrm{SW}_{\text {prä }}=18\right.$, $\mathrm{SW}_{\text {post }}=23$, Range: 0-30). Ihre Partnerschaftsqualität und -zufriedenheit stufte Frau Apfel im Partnerschaftsfragebogen konstant als hoch ein (PFB-K: SW $W_{\text {prä }}=$ $\left.24, \mathrm{SW}_{\text {post }}=27\right)$.

Die Patientin benötigte zur Bearbeitung der 8 Kernmodule durchschnittlich 11 Tage (Range: 3-33 Tage) und nahm 12 Tagebucheinträge vor. Der Kontakt $\mathrm{zu}$ ihrer Betreuerin umfasste 8 gesendete und beantwortete Nachrichten. Es erfolgte eine individuelle Terminabsprache, da die Patientin für die Bearbeitung der Module 6-8 mehr Zeit benötigte (Range: 11-33 Tage). Technische Schwierigkeiten wurden nicht berichtet.
Die Patientin gab an, sehr zufrieden mit der internetbasierten GPSPS-Therapie zu sein ( $\mathrm{SW}=3,8$; Range: $1-4$ ) [Boß et al., 2016]. Sie bewertete das Programm als qualitativ hochwertig und würde es einer Freundin weiterempfehlen, wenn diese ähnliche Hilfe benötige. Negative Therapieeffekte wurden nicht berichtet, die Patientin gab an, sich aufgrund des Programms besser zu fühlen.

Als wichtigste Wirkfaktoren nannte Frau Apfel im qualitativen Telefoninterview die intensive Beschäftigung mit sich und ihrem Körper sowie die regelmäßige Durchführung der schrittweisen Einführungsübungen. Sie beurteilte es als sehr hilfreich, dass das Behandlungsangebot explizit auf GPSPS zugeschnitten war. Durch das regelmäßige Training habe Sexualität außerdem einen größeren Stellenwert in ihrem Leben bekommen und sei infolgedessen ein Stück weit enttabuisiert worden. Letzteres habe sich darin gezeigt, dass sie zwei Freundinnen von ihren Beschwerden erzählt habe.

\section{Diskussion und Ausblick}

Die Fallauswertung zeigte, dass das Behandlungsprogramm Frauen auch mit lebenslangen GPSPS-Beschwerden unterstützen kann, Geschlechtsverkehr und damit assoziierte Therapieziele wie Schmerzbewältigung und Umgang mit negativen Gedanken zu erreichen, und in hoher Akzeptanz und Behandlungszufriedenheit resultieren kann.

Die vorgestellte Patientin profitierte insbesondere von der kleinschrittigen Zielsetzung der einzelnen Behandlungsschritte sowie den regelmäßigen Einführungsübungen mit Dilatoren. Die quantitative Auswertung zeigte neben der Fähigkeit zu Geschlechtsverkehr eine Abnahme der genito-pelvinen Schmerzen, der Angst vor Geschlechtsverkehr und der negativen penetrationsspezifischen Kognitionen. Diese Ergebnisse deuten darauf hin, dass das Behandlungsprogramm in allen Symptomdimensionen der GPSPS wirksam sein kann. Das Programm zeichnet sich durch seine Flexibilität aufgrund der zeit- und ortsunabhängigen Nutzbarkeit und Integration der Übungen in den Alltag aus und senkt durch anonyme Teilnahmemöglichkeit die Hemmschwelle einer Inanspruchnahme. Zu den weiteren Stärken des Programms zählt, dass Patientinnen die einzelnen Module in ihrem eigenen Tempo durchlaufen können und der Partner in die Übungen aktiv miteinbezogen wird. Das Fallbeispiel zeigt, dass Patientinnen für die Absolvierung des Behandlungsprogramms ausreichend Zeit einplanen sollten und ein zeitlich flexibler Bearbeitungszeitraum gegeben sein sollte (z.B. individuelle Anpassung der Erinnerungen an Modulabsolvierung). Als wichtig im Therapieverlauf ergab sich zudem, den Umgang mit potenziellen Rückschlägen zu thematisieren, indem auch Teilerfolge ausreichend validiert und Selbstwirksamkeitsstrategien zum Umgang mit Schwierigkeiten gefördert werden.

Eine Limitation dieser Kasuistik ist die bewusste Auswahl der Falldarstellung eines erfolgreichen Therapieverlaufs. Zudem beruhen die Therapieerfolgsmaße auf Selbstberichten ohne gynäkologische Fremdeinschätzung der Symptome. Vor Behandlungsbeginn ist jedoch der Ausschluss medizinisch behandelbarer GPSPS-Ursachen verpflichtend. Darüber hinaus sollte für die Erfassung von GPSPS in zukünftigen Studien ein Diagnostikinstrument entwickelt und validiert werden, das alle 4 Kardinalsymptome sowie den 
Leidensdruck und die Beeinträchtigungen, die mit der Symptomatik einhergehen, präzise erfasst. Zudem sollten zukünftige Studien den Therapieerfolg ebenfalls aus Sicht des Partners erheben.

Das internetbasierte Programm bietet die Möglichkeit, die Behandlung von GPSPS als begleitete Selbsthilfe oder Blended-CareKonzept, bei dem über Videokonferenz oder im Realsetting ein synchroner Therapeutenkontakt erfolgt, durchzuführen. Aus dem dargestellten Fallbericht kann geschlussfolgert werden, dass eine internetbasierte kognitiv-verhaltenstherapeutische Schmerz- und Sexualtherapie unter Einbezug des Partners für die Behandlung der GPSPS geeignet sein kann. Zeigen sich diese Therapieeffekte auch in der randomisiert-kontrollierten Wirksamkeitsstudie, kann das Programm eine evidenzbasierte flexible und niedrigschwellige Behandlungsmöglichkeit in der Gesundheitsversorgung für GPSPS darstellen und einen Beitrag zu einer flächendeckenderen Versorgung sexueller Funktionsstörungen in Deutschland leisten.

\section{Hinweis}

Zugang zum Behandlungsprogramm und das Betreuungsmanual können bei Anna-Carlotta Zarski (Anna-Carlotta.Zarski@fau.de) angefordert werden.

\section{Danksagung}

Das Projekt wurde durch die «Gender \& Diversity»-Förderung der Friedrich-Alexander-Universität Erlangen-Nürnberg gefördert. Ein besonderer Dank geht an Lina Bauer, Nadine Frank, Lennard Geiß, Kira Hauber, Kaja Kalinowski, Charlotte Kirchhoff und Johanna Standke, die an der Entwicklung des Behandlungsprogramms, der Implementierung und der Durchführung der Studie mit viel Engagement mitgewirkt haben.

\section{Disclosure Statement}

M.B. und D.D.E. sind Anteilseigner bei GET.ON Institut, dessen primäre Aufgabe der Transfer wissenschaftlich evaluierter internetbasierter Interventionen in die Gesundheitsversorgung darstellt.

\section{Literatur}

American Psychiatric Association: Diagnostic and Statistical Manual of Mental Disorders, ed 5. Washington, American Psychiatric Association, 2013.

Andersson E, Walén C, Hallberg J, Paxling B, Dahlin M, Almlöv J, et al: A randomized controlled trial of guided internet-delivered cognitive behavioral therapy for erectile dysfunction. J Sex Med 2011;8:2800-2809.

- Andrade L, Alonso J, Mneimneh Z, Wells J, Al-Hamzawi A, Borges G, et al: Barriers to mental health treatment: results from the WHO World Mental Health surveys. Psychol Med 2014;44:1303-1317.

Arnold LD, Bachmann GA, Rosen R, Kelly S, Rhoads GG: Vulvodynia: characteristics and associations with comorbidities and quality of life. Obstet Gynecol 2006; 107:617-624.

Bergeron S, Khalife S, Dupuis M-J, McDuff P: A randomized clinical trial comparing group cognitive-behavioral therapy and a topical steroid for women with dyspareunia. J Consult Clin Psychol 2016;84:259-268.

Boß L, Lehr D, Reis D, Vis C, Riper H, Berking M, et al: Reliability and validity of assessing user satisfaction with web-based health interventions. J Med Internet Res 2016;18:e234.

Brotto LA, Basson R, Smith KB, Driscoll M, Sadownik L: Mindfulness-based group therapy for women with provoked vestibulodynia. Mindfulness (N Y) 2015a;6: 417-432.

Brotto LA, Yong P, Smith KB, Sadownik LA: Impact of a multidisciplinary vulvodynia program on sexual functioning and dyspareunia. J Sex Med 2015b;12:238-247.

Carvalho J, Vieira AL, Nobre P: Latent structures of female sexual functioning. Arch Sex Behav 2012;41:907-917.

Cherner RA, Reissing ED: A comparative study of sexual function, behavior, and cognitions of women with lifelong vaginismus. Arch Sex Behav 2013;42:1605-1614.

Christensen BS, Grønbæk M, Osler M, Pedersen BV, Graugaard C, Frisch M: Sexual dysfunctions and difficulties in Denmark: prevalence and associated sociodemographic factors. Arch Sex Behav 2011;40:121-132.

Dillworth T, Jensen MP: The role of suggestions in hypnosis for chronic pain: a review of the literature. Open Pain J 2010;3:39-51.

Farmer MA, Meston CM: Predictors of genital pain in young women. Arch Sex Behav 2007;36:831-843.
Gagnier JJ, Riley D, Altman DG, Moher D, Sox H, Kienle GS; CARE Group: The CARE guidelines: consensusbased clinical case reporting guideline development. Dtsch Arztebl Int 2013;110:603-608.

Günzler C, Berner MM: Efficacy of psychosocial interventions in men and women with sexual dysfunctions - a systematic review of controlled clinical trials. Part 2 The efficacy of psychosocial interventions for female sexual dysfunction. J Sex Med 2012;9:3108-3125.

Jacobson E: Progressive Relaxation. Chicago, University of Chicago Press, 1938.

Khandker M, Brady SS, Vitonis AF, MacLehose RF, Stewart EG, Harlow BL: The influence of depression and anxiety on risk of adult onset vulvodynia. J Womens Health (Larchmt) 2011;20:1445-1451.

Klaassen M, Ter Kuile MM: Development and initital validation of the Vaginal Penetration Cognition Questionnaire (VPCQ) in a sample of women with vaginismus and dyspareunia. J Sex Med 2009;6:1617-1627.

Kliem S, Job A-K, Kröger C, Bodenmann G, Stöbel-Richter Y, Hahlweg K, et al: Entwicklung und Normierung einer Kurzform des Partnerschaftsfragebogens (PFBK) an einer repräsentativen deutschen Stichprobe. Z Klin Psychol Psychother 2012;41:81-89.

Ladwig I, Rief W, Nestoriuc Y: Welche Risiken und Nebenwirkungen hat Psychotherapie? - Entwicklung des Inventars zur Erfassung Negativer Effekte von Psychotherapie (INEP). Verhaltenstherapie 2014;24:252-263.

Latthe P, Latthe M, Say L, Gülmezoglu M, Khan KS: WHO systematic review of prevalence of chronic pelvic pain: a neglected reproductive health morbidity. BMC Public Health 2006;6:177.

Lester RA, Brotto LA, Sadownik LA: Provoked vestibulodynia and the health care implications of comorbid pain conditions. J Obstet Gynaecol Can 2015;37:9951005.

Masters W, Johnson V: Sex Therapy on Its 25th Anniversary: Why It Survives. St Louis, Masters \& Johnson Institute, 1986.

Moreira E, Brock G, Glasser D, Nicolosi A, Laumann E, Paik A, et al: Help-seeking behaviour for sexual problems: the global study of sexual attitudes and behaviors. Int J Clin Pract 2005;59:6-16.
Nguyen RH, Turner RM, Rydell SA, MacLehose RF, Harlow BL: Perceived stereotyping and seeking care for chronic vulvar pain. Pain Med 2013;14:1461-1467.

Pazmany E, Bergeron S, Van Oudenhove L, Verhaeghe J, Enzlin P: Body image and genital self-image in premenopausal women with dyspareunia. Arch Sex Behav 2013;42:999-1010

Peixoto MM, Nobre P: Prevalence and sociodemographic predictors of sexual problems in Portugal: a population-based study with women aged 18 to 79 years. J Sex Marital Ther 2015;41:169-180.

Reinecke A, Schöps D, Hoyer J: Sexuelle Dysfunktionen bei Patienten einer verhaltenstherapeutischen Hochschulambulanz: Häufigkeit, Erkennen, Behandlung. Verhaltenstherapie 2006;16:166-172.

Rosen R, Brown C, Heiman J, Leiblum S, Meston C, Shabsigh R, et al: The Female Sexual Function Index (FSFI): a multidimensional self-report instrument for the assessment of female sexual function. J Sex Marital Ther 2000;26:191-208.

Ter Kuile MM, Bulté I, Weijenborg PT, Beekman A, Melles $R$, Onghena P: Therapist-aided exposure for women with lifelong vaginismus: a replicated single-case design. J Consult Clin Psychol 2009;77:149-159.

Ter Kuile MM, De Groot HE, Tuijnman-Raasveld CC, Van Lankveld JJ: Therapist-aided exposure for women with lifelong vaginismus: a randomized waiting-list control trial of efficacy. J Consult Clin Psychol 2013;81:11271136

Van Lankveld JJ, ter Kuile MM, de Groot HE, Melles R, Nefs J, Zandbergen M: Cognitive-behavioral therapy for women with lifelong vaginismus: a randomized waiting-list controlled trial of efficacy. J Consult Clin Psychol 2006;74:168-178.

Vlaeyen JW, Linton SJ: Fear-avoidance and its consequences in chronic musculoskeletal pain: a state of the art. Pain 2000;85:317-332.

Wittchen H-U, Zaudig M, Fydrich T: Strukturiertes Klinisches Interview für DSM-IV. Göttingen, Hogrefe, 1997.

Zarski A-C, Rosenau C, Fackiner C, Berking M, Ebert DD: Internet-based guided self-help for vaginal penetration difficulties: results of a randomised controlled pilot trial. J Sex Med 2017;14:238-254. 